\title{
Monitoring dendritic cell and cytokine biomarkers during remission prior to relapse in patients with FLT3-ITD acute myeloid leukemia
}

\author{
Mareike Rickmann • Laura Macke • \\ Bala Sai Sundarasetty • Kathrin Stamer • \\ Constanca Figueiredo • Rainer Blasczyk • \\ Michael Heuser • Juergen Krauter • Arnold Ganser • \\ Renata Stripecke
}

Received: 15 February 2013 / Accepted: 25 March 2013 / Published online: 25 April 2013

(C) The Author(s) 2013. This article is published with open access at Springerlink.com

\begin{abstract}
Relapse occurs frequently after treatment of acute myeloid leukemia (AML) patients with the FMS-like tyrosine kinase 3-internal tandem duplication (ITD) mutation. The availability of immunologic biomarkers to predict patients at high risk could allow clinicians to accelerate alternative treatments such as stem cell transplantation, immunotherapy, or novel drugs. We have previously reported that first diagnostic (FD) ITD $^{+}$AML showed immunophenotypic and functional characteristics of arrested dendritic cell (DC) precursors. In this study, we show that the high frequency of precursor DCs in 16 FD ITD $^{+}$AML samples $\left(\mathrm{Lin}^{-} / \mathrm{HLA}-\mathrm{DR}^{+} / \mathrm{CD} 11 \mathrm{c}^{+} / \mathrm{CD} 123^{+}\right)$ was associated with a lack of terminal DCs (myeloid DCs: BDCA $-1^{+}$or BDCA $-3^{+}$; plasmacytoid DC: BDCA- $2^{+}$). We further evaluated prospectively the peripheral blood complete remission (CR) samples obtained from $11 \mathrm{ITD}^{+} \mathrm{AML}$ patients after chemotherapy regarding the frequency of DCs and their pattern of cytokine production. Whereas the aberrant frequencies of precursor and terminal plasmacytoid DCs resolved
\end{abstract}

Electronic supplementary material The online version of this article (doi:10.1007/s00277-013-1744-y) contains supplementary material, which is available to authorized users.

M. Rickmann · L. Macke • B. S. Sundarasetty $\cdot$ K. Stamer •

M. Heuser $\cdot$ J. Krauter $\cdot$ A. Ganser $\cdot$ R. Stripecke $(\triangle)$

Hematology, Hemostasis, Oncology

and Stem Cell Transplantation,

Hannover Medical School (MHH),

OE 6860, Carl-Neuberg Str. 1,

30625 Hannover, Germany

e-mail: Stripecke.Renata@mh-hannover.de

C. Figueiredo $\cdot$ R. Blasczyk

Department of Transfusion Medicine, MHH,

Hannover, Germany during remission, the myeloid DC compartment did not fully recover. For an available cohort of patients $(n=4)$ who could be monitored over a period of $>15$ months after FD, we identified IL-10, TNF- $\alpha$, IL- 6 , and IL-1 $\beta$ as cytokines produced by the CR samples at high levels a few months prior to relapse. Cell-free supernatant of an FD ITD $^{+}$AML sample stimulated monocytes obtained from two healthy donors to secrete IL-10, TNF- $\alpha$, IL-6, and IL-1 $\beta$. Thus, we hypothesize that $\mathrm{ITD}^{+}$AML minimal residual disease can act directly as dysfunctional antigen-presenting cells or indirectly by production of factors that convert monocytes into myeloid-derived suppressor cells secreting cytokines that promote immune evasion. Monitoring these immunologic biomarkers could improve prediction of relapse.

Keywords Leukemia · Dendritic cell · Immune monitoring · Myeloid-derived suppressor cells · Risk of relapse · Inflammatory cytokines

\section{Introduction}

Acute myeloid leukemia (AML) is a very heterogeneous disease and risk stratification has recently been improved by detection of genetic mutations in samples collected at first diagnosis (FD) and complete remission (CR) [1]. One of the most common mutations in AML occurs in the open reading frame of FMS-like tyrosine kinase 3 (FLT3), which is the receptor for FLT3-ligand, a cytokine with pivotal function in dendritic cell (DC) differentiation and in stem cell renewal $[2,3]$. The mutations in the FLT3 receptor occur by point mutations in the kinase domain or by internal tandem 
duplications (ITD) of the juxtamembrane or tyrosine kinase domain. These mutations afflict approximately $30 \%$ of the newly diagnosed AML cases corresponding to a group of patients at high risk $[4,5]$. Once FLT3-ITD is identified, mutations in other loci can be used to aid in the outcome prediction (such as NPM1, MLL, N-RAS, and WT1) [5-7]. Novel sensitive approaches to detect leukemia relapse through next-generation sequencing of mutated hotspots such as FLT3-ITD and NPM1 are currently in development [8].

Clinical evidence indicates that allogeneic stem cell transplantation (SCT) of $\mathrm{ITD}^{+} \mathrm{AML}$ patients results in the best survival outcome and is the routine clinical practice for eligible patients [9]. The mechanistic effects of allo-SCT are believed to be largely immunological, due to the capacity of $\mathrm{T}$ cells from the donor to mount a curative and long-term graft-versus-leukemia effect against minimal residual disease (MRD) [10]. Unfortunately, older patients and patients without suitable donors are not eligible for allo-SCT and conventional chemotherapy is the standard of care. For these patients, novel therapies in clinical development such as immunotherapy or novel drugs are certainly warranted. In addition, novel diagnostic approaches such as immune monitoring would potentially impact in the prediction of MRD immune evasion and relapse.

Dendritic cells play a key role in immune regulation and function [11, 12]. The DC compartment in cancer patients is often deregulated, resulting in the accumulation of immature and/or dysfunctional DCs [13]. We have recently shown that FD samples of adult ITD ${ }^{+}$AML patients contain an aberrant accumulation of cells with immunophenotypic and functional characteristics of arrested DCs [14]. Thus, we hypothesize that residual leukemic $\mathrm{ITD}^{+}$DCs could potentially affect the differentiation of normal DCs of patients during disease recovery through paracrine mechanisms. In that regard, we evaluated if cytokines (IL-10, TNF- $\alpha$, IL-6, and IL-1 $\beta$ ) proposed by several groups as indicators of a broad range of immunopathological conditions affecting cancer [15-20] could also serve as potential biomarkers to predict $\mathrm{ITD}^{+}$leukemia relapse.

In this study, we prospectively collected samples from 11 $\mathrm{ITD}^{+}$AML patients treated with standard chemotherapy. Cohorts of nonresponders, patients who entered remission and then relapsed or patients with relapse-free survival for $>15$ months, were identified. Peripheral blood samples of ITD $^{-}$AML patients and healthy donors (HD) were used as control groups. Kinetic analyses of four ITD $^{+}$AML patients who entered CR but then relapsed between 16 and 24 months after FD showed a consistent pattern of arrested terminal differentiation of myeloid DCs and upregulation of IL-10 (an anti-inflammatory cytokine), TNF- $\alpha$, IL-6, and IL-1 $\beta$ (pro-inflammatory cytokines) months prior to leukemia relapse. We provided experimental evidence that soluble factors produced by $\mathrm{ITD}^{+}$AML stimulated monocytes from healthy donors to produce IL-10, TNF- $\alpha$, IL-6, and IL- $1 \beta$.

\section{Materials and methods}

\section{Patient samples}

This study was performed in accordance with the declaration of Helsinki and was approved by the local ethics committee of the Hannover Medical School. Peripheral blood samples from adult (18-83 years) AML patients were collected after written informed consent. Twenty-six ITD ${ }^{+}$and 28 ITD $^{-}$patients, for whom FD samples were available, were included in this study (Suppl. Table 1). Peripheral blood mononuclear cells (PBMCs) were collected from $26 \mathrm{ITD}^{+}$patients for up to 24 months after FD and they were grouped according to the clinical outcome (Suppl. Fig. 1b and Table 1): nonresponders (NR, no CR after induction therapy, i.e., $>5 \%$ blasts in the $\mathrm{BM})$ and CR. CR patients were split between those who eventually relapsed $>15$ months after FD (CR/REL) and those patients with disease-free survival (CR/DFS, no relapse up to 2 years after FD).

\section{Cytogenetics, FLT3-ITD, and WT1 analyses}

Cytogenetic and molecular genetic studies (Table 1 and Suppl. Table 1) were performed by the German-Austrian Acute Myeloid Leukemia Study Group at Hannover Medical School or at the University of Ulm. Blood diagnostic samples were analyzed for the presence of the ITD mutations in the FLT3 gene by polymerase chain reaction as described previously [21]. The upregulation of WT1 mRNA levels was used as a putative approach to predict relapse as previously described [22]. RNA was extracted from approximately $5 \times 10^{6} \mathrm{PBMCs}$ using the Qiagen RNeasy kit according to the protocol of the manufacturer (Qiagen, Hilden, Germany). Real-time quantitative polymerase chain reaction (RQ-PCR) was performed with extracted RNA using the WT1 ProfileQuant ${ }^{\mathbb{B}}$-Kit (ELN) (Ref: PQPP-02-CE) from Ipsogen (Luminy Biotech Enterprises, Marseille, France). The RQ-PCR consists of two holding stages of 1 cycle each at $50{ }^{\circ} \mathrm{C}$ for $2 \mathrm{~min}$ followed by $95^{\circ} \mathrm{C}$ for $10 \mathrm{~min}$ and a cycling stage at $95^{\circ} \mathrm{C}$ for $15 \mathrm{~s}$ and $60^{\circ} \mathrm{C}$ for 1 min (50 cycles) in a StepOnePlus Real-Time PCR system (Applied Biosystems, Darmstadt, Germany). Standard curves and fluorescence calculation were performed using the StepOnePlus Real-Time PCR system and Microsoft Excel (Microsoft, Germany).

Immunophenotypic analyses of precursor and terminal DCs

PBMCs obtained from patients and healthy volunteers were isolated by standard density gradient centrifugation using Ficoll (Biocoll separating solution, Greiner, Bio-One, Germany) separation and cryopreserved in $90 \%$ FBS and $10 \%$ DMSO. Progenitor DCs were identified using a commercially available kit ("Peripheral Blood Dendritic Cell 
Table 1 Patient characteristics of $\mathrm{ITD}^{+}$patient groups NR, CR/ REL, and CR/DFS
$N R$ nonresponders, $C R / R E L$ complete remission followed by relapse (within 2 years from first diagnosis), $C R / D F S$ complete remission and disease-free survival (for $>2$ years from first diagnosis), $F A B$ French American British Classification, $W B C$ white blood cells $\times 1,000 / \mu 1$, NPM1 mutation exon 12 nucleophosmin mutation, WT1 Wilm's tumor 1 gene mutation, $R E L$ relapse, $n / a$ not available

${ }^{a}$ Patients analyzed for kinetics of late leukemia relapse and correlation with DC and cytokine profile

\begin{tabular}{|c|c|c|c|c|c|c|c|c|c|}
\hline \multirow[t]{2}{*}{ ID } & \multirow[t]{2}{*}{ Sex } & \multirow[t]{2}{*}{ FAB } & \multirow[t]{2}{*}{ WBC } & \multirow[t]{2}{*}{ Age } & \multirow[t]{2}{*}{ Karyotype } & \multicolumn{3}{|l|}{ Mutations } & \multirow[t]{2}{*}{ REL (month) } \\
\hline & & & & & & FLT3 ITD & NPM1 & WT1 & \\
\hline \multicolumn{10}{|l|}{ NR } \\
\hline$\# 1$ & $\mathrm{~F}$ & M4 & 1.4 & 57 & 46, XX & + & - & + & - \\
\hline$\# 2$ & M & M5 & $\mathrm{n} / \mathrm{a}$ & 45 & Complex & + & - & + & - \\
\hline$\# 3$ & M & M5 & 119.2 & 57 & $\mathrm{n} / \mathrm{a}$ & + & - & - & - \\
\hline$\# 4$ & M & sAML & 259 & 67 & $\mathrm{n} / \mathrm{a}$ & + & - & $\mathrm{n} / \mathrm{a}$ & - \\
\hline$\# 5$ & M & $\mathrm{n} / \mathrm{a}$ & 97 & 83 & $\mathrm{n} / \mathrm{a}$ & + & + & + & - \\
\hline \multicolumn{10}{|c|}{$\mathrm{CR} / \mathrm{REL}$} \\
\hline$\# 6^{\mathrm{a}}$ & M & M4 & 110.1 & 40 & $46, X Y$ & + & + & + & 18 \\
\hline$\# 7^{\mathrm{a}}$ & $\mathrm{F}$ & M4 & 98.1 & 53 & 46, XX & + & + & + & 16 \\
\hline$\# 8^{a}$ & $\mathrm{~F}$ & M5 & 66.7 & 48 & Complex & + & - & + & 20 \\
\hline$\# 9^{\mathrm{a}}$ & $\mathrm{F}$ & M2 & 69.9 & 52 & $46, \mathrm{XX}$ & + & + & + & 24 \\
\hline$\# 10$ & M & M5a & 56.2 & 44 & 46, XY & + & + & $\mathrm{n} / \mathrm{a}$ & 10 \\
\hline$\# 11$ & M & M4 & 9 & 24 & $46, X Y$ & + & - & + & 3 \\
\hline$\# 12$ & $\mathrm{~F}$ & M4 & 0.4 & 78 & Complex & + & - & + & 3 \\
\hline$\# 13$ & $\mathrm{~F}$ & M5a & 107 & 53 & $46, X X$ & + & + & $\mathrm{n} / \mathrm{a}$ & 8 \\
\hline \multicolumn{10}{|c|}{ CR/DFS } \\
\hline$\# 14$ & $\mathrm{~F}$ & M4 & 6.3 & 64 & 46, XX & + & + & + & No REL \\
\hline$\# 15$ & $\mathrm{~F}$ & M1 & $\mathrm{n} / \mathrm{a}$ & 66 & 46, XX & + & + & + & No REL \\
\hline$\# 16$ & M & M4 & 3.1 & 77 & 46, XY & + & + & + & No REL \\
\hline$\# 17$ & M & $\mathrm{n} / \mathrm{a}$ & $\mathrm{n} / \mathrm{a}$ & 32 & $\mathrm{n} / \mathrm{a}$ & + & - & $\mathrm{n} / \mathrm{a}$ & No REL \\
\hline$\# 18$ & $\mathrm{~F}$ & M2 & 243 & 70 & 46, XX & + & + & $\mathrm{n} / \mathrm{a}$ & No REL \\
\hline$\# 19$ & M & $\mathrm{n} / \mathrm{a}$ & $\mathrm{n} / \mathrm{a}$ & 40 & $\mathrm{n} / \mathrm{a}$ & + & + & $\mathrm{n} / \mathrm{a}$ & No REL \\
\hline$\# 20$ & $\mathrm{~F}$ & M4 & 45.2 & 63 & 46, XX & + & + & + & No REL \\
\hline$\# 21$ & $\mathrm{~F}$ & M1 & 3.6 & 50 & 46, XX & + & + & + & No REL \\
\hline$\# 22$ & M & $\mathrm{n} / \mathrm{a}$ & 12 & 40 & 46, XY & + & - & + & No REL \\
\hline$\# 23$ & $\mathrm{~F}$ & M3 & $\mathrm{n} / \mathrm{a}$ & 65 & APL & + & - & $\mathrm{n} / \mathrm{a}$ & No REL \\
\hline$\# 24$ & $\mathrm{~F}$ & M5 & $\mathrm{n} / \mathrm{a}$ & 61 & $46, \mathrm{XX}$ & + & - & $\mathrm{n} / \mathrm{a}$ & No REL \\
\hline$\# 25$ & M & M6 & 5.9 & 18 & 46, XY & + & + & $\mathrm{n} / \mathrm{a}$ & No REL \\
\hline$\# 26$ & M & M5b & $\mathrm{n} / \mathrm{a}$ & 67 & 46, XY & + & + & $\mathrm{n} / \mathrm{a}$ & No REL \\
\hline
\end{tabular}

Detection," Becton Dickinson BD, San Jose, CA, USA). The protocol is based on a four-color staining. For the detection of myeloid and plasmacytoid progenitor DCs, we used lineage cocktail 1 (FITC) containing monoclonal antibodies (mABs) against CD3, CD14, CD16, CD19, $\mathrm{CD} 20$, and $\mathrm{CD} 56$ as a negative selection, a $\mathrm{mAB}$ against HLA-DR (PerCp, clone L243), a mAB against the CD11c myeloid DC marker (APC, clone S-HCL-3), and a mAB against the CD123 plasmacytoid DC marker (PE, clone 95F). Fifty thousand viable cells gated on the forward scatter (FSC)/side scatter (SSC) were negatively selected using the lineage markers. The resulting $\mathrm{Lin}^{-}$population was analyzed for HLA-DR/CD11c ("precursor myeloid dendritic cells (mDCs)") or HLA-DR/CD123 ("precursor plasmacytoid dendritic cells (pDCs)"). Terminal DCs were identified using a commercially available kit ("Blood Dendritic Cell Enumeration," Miltenyi Biotec, BergischGladbach, Germany). The protocol is based on a four- color staining: for $\mathrm{mDC1} 1$, a $\mathrm{mAB}$ against $\mathrm{BDCA}-1$ (CD1c, PE); for pDC-, a mAB against BDCA-2 (CD303, FITC); and for $\mathrm{mDC} 2$ detection, a $\mathrm{mAB}$ against BDCA-3 (CD141, APC). We used a photoaffinity fluorescent "dead cell discriminator" (PE-Cy5) and mABs against CD19 and CD14 to (PE-Cy5) to exclude B cells, monocytes, granulocytes, and dead cells. Fifty thousand viable cells gated on the FSC/SSC scatter and excluding dead cells and $\mathrm{CD} 19^{+} / \mathrm{CD} 14^{+}$cells were analyzed for expression of BDCA-1 (mDC1), BDCA-2 (pDC), and BDCA-3 $(\mathrm{mDC} 2)$. Stained cells were analyzed with a FACSCalibur using CellQuest software (BD, San Jose, CA, USA).

Analyses of cytokine secretion

Cell-free supernatants of PBMC samples obtained from leukemia patients were obtained by seeding of $1 \times 10^{6}$ viable cells in $1 \mathrm{ml}$ of X-vivo medium (Lonza, Belgium) in 12-well 
Fig. 1 FD samples of ITD $^{+}$

AML patients $(n=16)$ showed a more pronounced alteration of the dendritic cell pattern in comparison with the FD samples of ITD ${ }^{-}$AML patients $(n=22)$. a Frequencies of precursor DCs. b Frequencies of terminal DCs. Single asterisk: significantly higher than HD $(p<0.05)$. Double asterisk: significantly lower than HD $(p<0.05)$. [The dotted line is showing the average values for $\mathrm{HD}$ ]

\section{A) Analyses of DC precursors at FD}
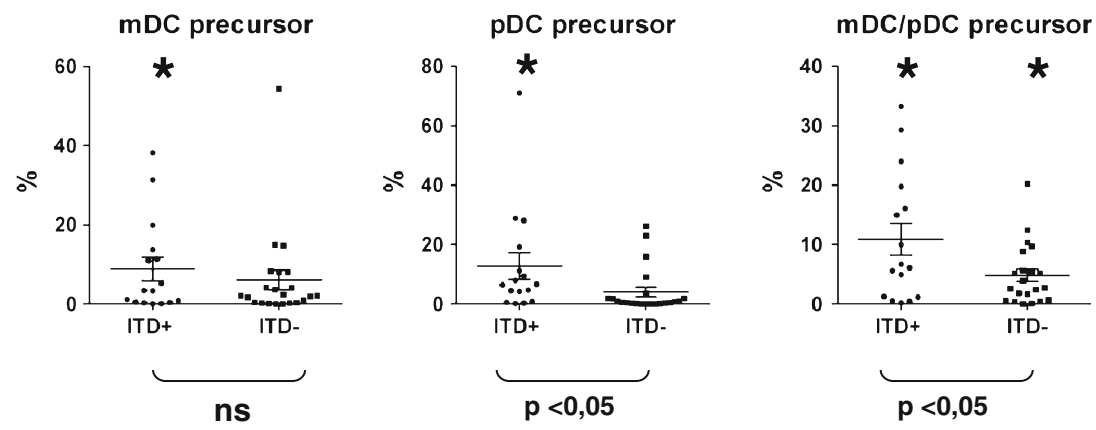

B) Analyses of terminally differentiated DCs at FD
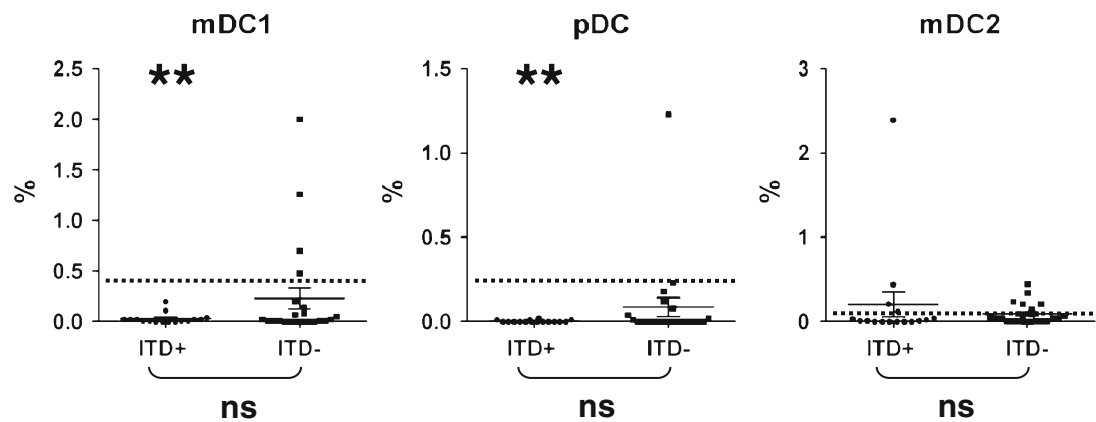

plates (TPP, Switzerland), incubated for $24 \mathrm{~h}$ at $37{ }^{\circ} \mathrm{C}$ and $5 \% \mathrm{CO}_{2}$, collected, centrifuged to remove cells and debris, and cryopreserved at $-80{ }^{\circ} \mathrm{C}$. Luminex ${ }^{\circledR}$ analyses were performed with a Cytokine Human 14-Plex Panel (Millipore, Schwalbach, Germany) for analyses of IL-10, TNF- $\alpha$, IL-6, and IL- $1 \beta$. As controls, supernatants obtained from $\mathrm{PB}$ samples obtained from four healthy donors were included in the analyses. Cytokine standards supplied by the manufacturer were run on each plate together with the test samples. Samples were treated accordingly to manufacturer's instructions. For cytokine detection, data were acquired on a Luminex-200 System and analyzed with the Xponent software v.3.0 (Invitrogen).

Treatment of $\mathrm{CD} 14^{+}$monocytes with AML supernatant

The cell culture supernatant collected from one of the FD samples in the study (CR/REL patient \#6) as described above was filtered with $0.2 \mu \mathrm{M}$ and diluted with X-vivo medium at dilutions of $1: 3$ and 1:9. $\mathrm{CD} 14^{+}$monocytes were isolated from the leukapheresis of healthy donors using CD14 microbeads (Miltenyi, Germany). The isolated $\mathrm{CD}_{1} 4^{+}$monocytes were seeded at a density of $5 \times 10^{6}$ cells per well and were cultured in X-vivo medium or with diluted 2-ml AML supernatants in a six-well plate. The plates were incubated at $37{ }^{\circ} \mathrm{C}$ for 3 days. At the end of the incubation period, the cell viability and monocyte purity were assessed by flow cytometric analyses of 7AAD and CD14. The cell supernatants were collected and the cytokine profile was analyzed by Luminex ${ }^{\circledR}$ as described above.

\section{Results}

FD samples of $\mathrm{ITD}^{+}$AML patients showed a more pronounced alteration of the dendritic cell pattern in comparison to FD samples of ITD $^{-}$AML patients

We had previously reported that FD samples of ITD $^{+}$patients presented with an accumulation of $\mathrm{Lin}^{-} / \mathrm{HLA}-\mathrm{DR}^{+}$ cells that co-expressed the DC markers CD11c and/or CD123. These leukemic DCs contained ITD mutations and could be driven to differentiate into mature mDCs or pDCs in vitro upon culture with cytokines, but they were not fully capable of secreting several cytokines [14]. We therefore deduced that $\mathrm{CD} 11 \mathrm{c}^{+}, \mathrm{CD} 123^{+}$, and $\mathrm{CD} 11 \mathrm{c}^{+} / \mathrm{CD} 123^{+}$leukemic DCs were arrested in the dendritic cell differentiation pathway and that $\mathrm{CD} 11 \mathrm{c}^{+}$and $\mathrm{CD} 123^{+}$should be rather considered as DC precursor markers. In order to assess the frequencies of mature DCs in leukemia samples, we therefore used a second immunophenotypic panel. Here, terminal DCs were defined as mDC1 $\left(\right.$ BDCA- $\left.1^{+}\right)$, $\mathrm{pDC}\left(\mathrm{BDCA}^{-} 2^{+}\right)$, or $\mathrm{mDC} 2\left(\mathrm{BDCA}-3^{+}\right)$. Validation of the immunophenotypic analyses was performed with cryopreserved/thawed PBMC 
obtained from nine healthy control donors. For both types of immunophenotypic analyses, we observed a higher frequency of mDCs (approximately $0.4 \%$ ) compared to pDCs (approximately $0.2 \%$ ) (Suppl. Fig. 1a). The frequencies of mixed lineage $\mathrm{mDC} / \mathrm{pDCs}\left(\mathrm{CD} 123^{+} / \mathrm{CD} 11 \mathrm{c}^{+}\right)$and of $\mathrm{mDC} 2$ were quite low (below $0.1 \%$ ). Using the same staining procedures, we examined FD-cryopreserved samples of $\mathrm{ITD}^{+}$and ITD $^{-}$patients who were diagnosed and treated at the Hannover Medical School. We compared the FD samples of $16 \mathrm{ITD}^{+}$and $22 \mathrm{ITD}^{-}$patients, who eventually entered CR. The cohorts were reasonably similar in terms of gender distribution, age, WBC counts, NPM1 mutation frequency, FAB types, and cytogenetics (Suppl. Table 1). In contrast to the homogeneous frequencies of DCs in PBMC samples obtained from HD (0-0.83\%), FD samples from $\mathrm{ITD}^{+}$and $\mathrm{ITD}^{-}$patients showed a high variability in the frequencies of precursor DCs, ranging from 0 to $71 \%$ (Fig. 1a and Suppl. Figs. 1a and 2a). We observed the previously reported accumulation of $\mathrm{mDC} / \mathrm{pDC}$ precursors $\left(\mathrm{CD} 123^{+} / \mathrm{CD}_{\left.11 \mathrm{c}^{+}\right)}\right.$in $\mathrm{FD}$ obtained from $\mathrm{ITD}^{+}$patients (Fig. 1a and Suppl. Fig. 2a). The frequencies of $\mathrm{mDC} / \mathrm{pDC}$ precursors were significantly increased in both $\operatorname{ITD}^{+}(p=$ $0.0065)$ and $\operatorname{ITD}^{-}(p=0.0077)$ FD patient samples compared to HD PBMCs. Notably, the accumulation of $\mathrm{mDC} / \mathrm{pDC}$ precursors was significantly higher in the FD of $\mathrm{ITD}^{+}$patients compared to the FD of ITD $^{-}$patients (mDC: $p=0.046$; pDC: $p=0.049)$. The same samples demonstrated almost complete lack of terminal mDC1s $\left(\mathrm{BDCA}-1^{+}\right)$and $\mathrm{pDCs}$ $\left(\right.$ BDCA-2 ${ }^{+}$) (Fig. 1b and Suppl. Fig. 2b). Of note, significantly lower frequencies of terminal mDC1 $(p<0.0001)$ and pDC $(p<0.0001)$ were found in FD samples of ITD $^{+}$patients compared to HD, whereas the frequencies of terminal DCs in FD samples of ITD ${ }^{-}$were not significantly affected (Fig. 1b). Thus, these results fully corroborated with our previous findings using another AML cohort, showing that the ITD mutation in AML is correlated with the accumulation of $\mathrm{mDC} / \mathrm{pDC}$ precursors. Furthermore, these leukemic ITD $^{+}$DC precursors did not display the terminally differentiated DC immunophenotype. These findings combined underscore the notation that $\mathrm{ITD}^{+}$AML corresponds to an arrested DC lineage malignancy.

FD samples obtained from ITD ${ }^{+}$NR patients secreted higher levels of IL-10 and stress cytokines in vitro in comparison to $\mathrm{CR}$ patients

IL-10 is produced mostly by macrophages and myeloid DCs and is generally regarded as an anti-inflammatory cytokine exerting several effects in immunomodulation of the cancer microenvironment and cancer progression [17]. Direct inhibition of myeloid DC differentiation mediated through IL10 and IL-6 was proposed to induce a potentially immunocompromising microenvironment in cancer patients
[23]. IL- 6 , TNF- $\alpha$, and IL-1 $\beta$ are predominantly secreted by activated monocytes and macrophages and play a central role in innate inflammatory events. Remarkably, the neuroendocrine stress system in human beings is also triggered via soluble factors such as IL-6, TNF- $\alpha$, and IL- $1 \beta$, heretofore named "stress cytokines" $[17,24]$. Therefore, we proposed to evaluate whether FD containing ITD $^{+}$leukemic cells would show particular patterns of expression of IL-10 and/or stress cytokines. FD samples obtained from NR patients showed higher levels of production of all stress cytokines compared with PBMC of HD, whereas IL-10 levels were similar (Fig. 2). On the other hand, FD samples obtained from $\mathrm{ITD}^{+} \mathrm{CR}$ patients (CR/REL and CR/DFS) showed lower levels of secreted IL-10, TNF- $\alpha$, IL-6, and IL-1 $\beta$ than PBMCs of HD controls (Fig. 2). Thus, compared with the DC immunophenotypic analyses of $\mathrm{ITD}^{+} \mathrm{AML}$ showing a more uniform pattern, the cytokine secretion pattern was more heterogeneous. These findings suggested that either the ITD ${ }^{+}$leukemic cells from NR patients secreted cytokines in a different pattern than cells obtained from $\mathrm{CR} / \mathrm{REL}$ and $\mathrm{CR} / \mathrm{DFS}$, or that bystander cells contained in FD samples of NR patients could be responsible for the secretion of the cytokines.

CR samples obtained from ITD $^{+}$AML patients showed lower recovery of myeloid DCs compared with samples obtained from ITD $^{-}$AML patients

Low DC frequencies in leukemia patients after initial treatment and/or HSCT have been associated with relapse and a poor outcome $[25,26]$. Therefore, we compared the frequencies of precursor and terminal DCs in samples obtained from $\mathrm{ITD}^{+}(n=11)$ and $\mathrm{ITD}^{-}(n=13)$ patients in CR (Suppl. Fig. $1 \mathrm{~b}$ and Table 1). During $\mathrm{CR}$, the $\mathrm{mDC} / \mathrm{pDC}$ precursor population disappeared in both the $\mathrm{ITD}^{+}$and $\mathrm{ITD}^{-}$cohorts (Fig. 3a). ITD ${ }^{+}$CR patients showed a trend towards lower frequencies of terminal myeloid DCs (mDC1, $p=0.057$; mDC2, $p=0.0912$ ) in comparison to HD (Fig. 3b). These findings suggested that the aberrant DC patterns observed at $\mathrm{ITD}^{+}$AML diagnosis tended to normalize during $\mathrm{CR}$, but did not return to the normal situation observed in healthy individuals. Thus, despite of this modest alteration in DC frequency, we could not identify a major aberration in $\mathrm{CR}$ samples of ITD $^{+}$AML patients as a whole.

Kinetic analyses of CR samples of $\mathrm{ITD}^{+}$patients showed persistent accumulation of $\mathrm{mDC} / \mathrm{pDC}$ precursors and a decline in the levels of terminal mDCs who relapsed after 15 months

We chose four patients who experienced leukemia relapse within 2 years after FD (see Table 1, CR/REL patients 6, 7, 8 , and 9) for performing kinetic analyses. PBMC samples 
Fig. 2 FD samples obtained from NR ITD ${ }^{+}$AML patients $(n=4)$ secreted higher levels of stress cytokines in vitro in comparison with patients who entered CR $(n=7) . N R$

nonresponders $(n=4), C R / R E L$ complete remission followed by relapse ( $<2$ years; $n=4)$, $C R / D F S$ complete remission and disease-free survival ( $>2$ years) $(n=3), H D$ healthy donors $(n=4)$. Single asterisk: significantly lower than HD $p<0.05$. [Detection level for cytokines is $3.2 \mathrm{pg} / \mathrm{ml}$ ]
IL-10

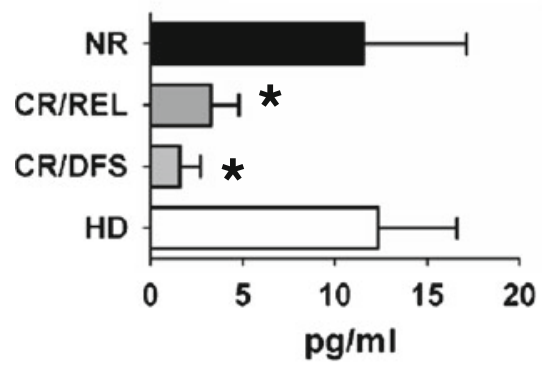

IL-6

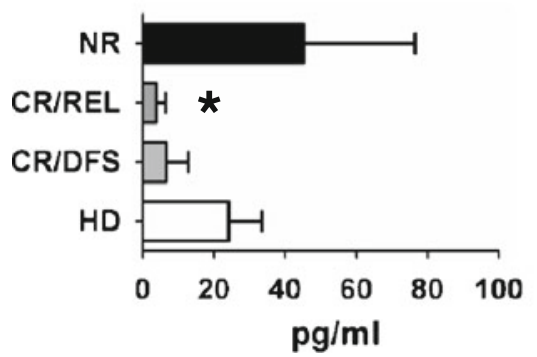

TNF-alpha

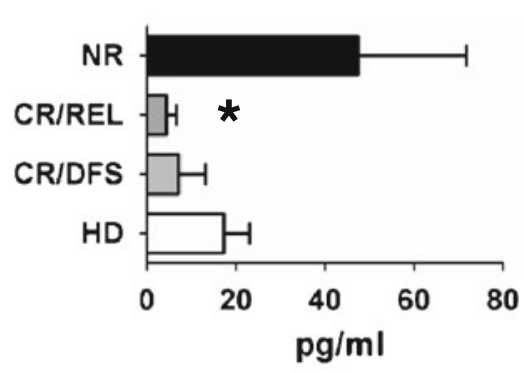

IL-1beta

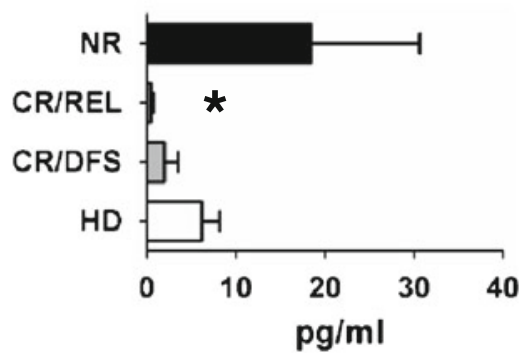

were collected five times sequentially after FD (every 3 months and up for 15 months). The frequencies of precursor $\mathrm{mDCs}$ and pDCs normalized during the first 6 months of CR (although always lower than healthy controls), but then decreased continuously until 15 months (Fig. 4a). The mixed lineage $\mathrm{mDC} / \mathrm{pDC}$ population drastically decreased
Fig. 3 CR samples obtained from $\mathrm{ITD}^{+}$AML patients $(n=$ 11) showed suboptimal recovery of myeloid DCs compared with samples obtained from ITD $^{-}$AML patients $(n=13)$. a

Normalization in the frequencies of precursor DCs. b Trend for lower frequencies of terminal mDCs. [The dotted line is showing the average values for $\mathrm{HD}$ ]

\section{A) Analyses of DC precursors at CR}
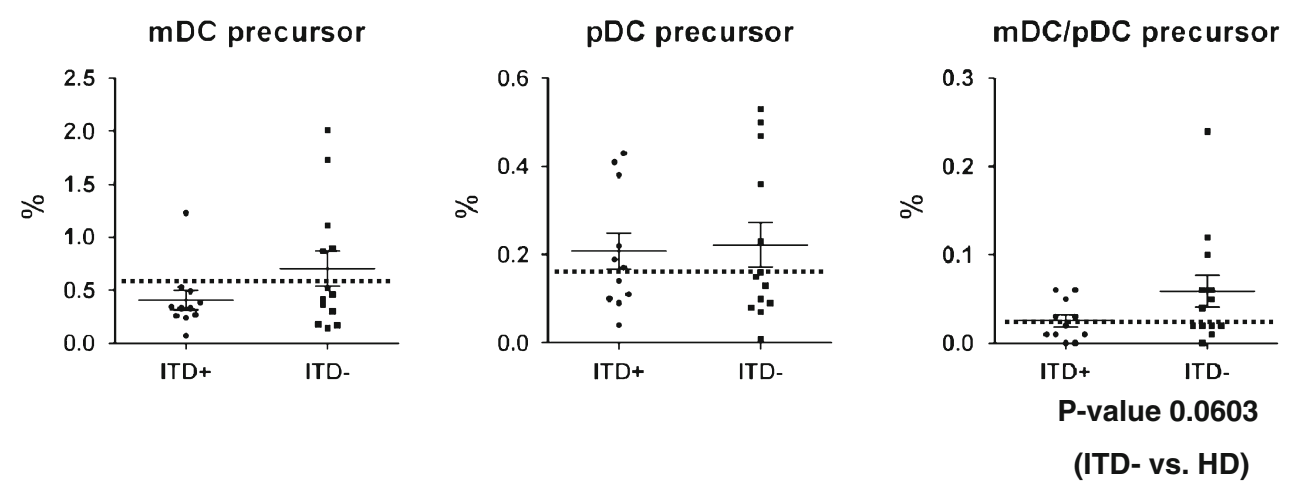

\section{B) Analyses of terminally differentiated DCs at CR}

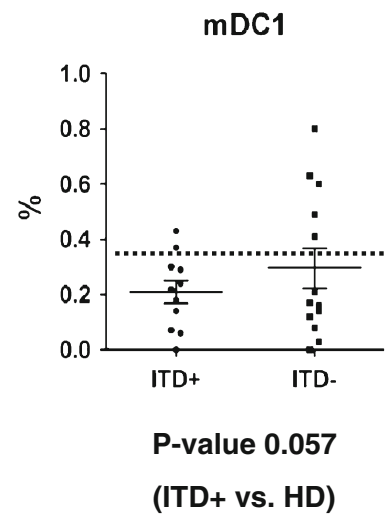

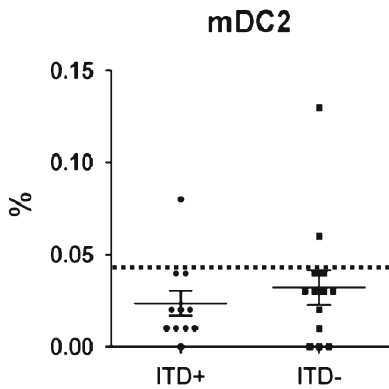

P-value 0.0912

(ITD+ vs. HD) 
during CR, but was nevertheless still detectable throughout the observation period (Fig. 4a). The frequencies of terminal mDC1s were always lower compared to HD controls, whereas terminal $\mathrm{mDC} 2 \mathrm{~s}$ returned to normal levels at 15 months (Fig. 4b). The frequencies of terminal pDCs did not reveal significant fluctuations during CR (Fig. 4b). Altogether, these results showed that for patients who relapsed, alterations in $\mathrm{mDC}$ frequency persisted throughout the remission period. Notably, both precursor and terminally differentiated $\mathrm{mDC}$ tended to disappear prior to relapse.

IL-10 and stress cytokines secreted by CR/REL samples were observed as a later event, preceding relapse

We had observed that secretion of IL-10, TNF- $\alpha$, IL-6, and IL-1 $\beta$ cytokines in FD samples of ITD $^{+}$nonresponder patients was higher than for patients who entered CR (Fig. 2), but we could not discern if the leukemic cells or possible bystander cells were producing the cytokines. IL-10 and stress cytokines have been linked with the occurrence of myeloid-derived suppressor cells (MDSCs), which can hinder DC development from myeloid precursor cells [27-29]. Since CR leukemic cells were not detectable in PBMC samples by morphology, we investigated if non-leukemic cells contained in the PBMC samples might lead to aberrant cytokine production. We used samples from the same four CR/REL patients described above for the cytokine secretion patterns at earlier (6 months, Fig. 5a) and later time points
(12 months, Fig. 5b) after FD. As controls, we included samples obtained from three CR/DFS patients and four HD in the analyses. At 6 months after FD, IL-10 secretion by PBMCs of CR/REL samples was significantly lower than for samples from CR/DFS patients and HD, but the levels of the stress cytokines were comparable (Fig. 5a). Yet, at 12 months after FD, there was a trend for higher production of all stress cytokines in CR/REL samples in comparison with CR/DFS patients and HD samples (Fig. 5b). Nevertheless, results correlated with the lower frequencies of $\mathrm{mDCs}$ observed in CR/REL patients 12 months after FD (Fig. 4) suggesting that increased production of stress cytokines by cells contained in the PBMC samples was linked to decreased $\mathrm{mDC}$ differentiation. Since the PBMC samples at 12 months did not show detectable leukemic blasts, this finding underscored our hypothesis that other cells, such as myeloid precursor cells or myeloid-derived suppressor cells, could be implicated in the secretion of the cytokines.

Soluble factors produced by the $\mathrm{ITD}^{+} \mathrm{FD}$ samples can increase viability and stimulate monocytes to secrete IL-10 and stress cytokines

It was previously shown that cell supernatants obtained from leukemic cells inhibited DC differentiation ex vivo, promoted by recombinant cytokines [30]. We set up similar assays with cell culture supernatants obtained from an $\mathrm{ITD}^{+}$ FD sample and from a leukemic cell line. However, we did
Fig. 4 Kinetic analyses of DC patterns during $\mathrm{CR}$ of $\mathrm{ITD}^{+}$ AML patients $(n=4)$ showed persistent accumulation of $\mathrm{mDC} / \mathrm{pDC}$ precursors and a decline in the levels of terminal $\mathrm{mDC} 1$ prior to relapse. a Frequencies of precursor DCs at $0,3,6,9,12$, and 15 months. b Frequencies of terminal DCs at $0,3,6,9,12$, and 15 months. Single asterisk: significantly higher than HD $(p<0.05)$. Double asterisk: significantly lower than HD $(p<0.05)$. [The dotted line is showing the average values for $\mathrm{HD}$ ]

\section{A) Analyses of DC precursors prior to relapse}
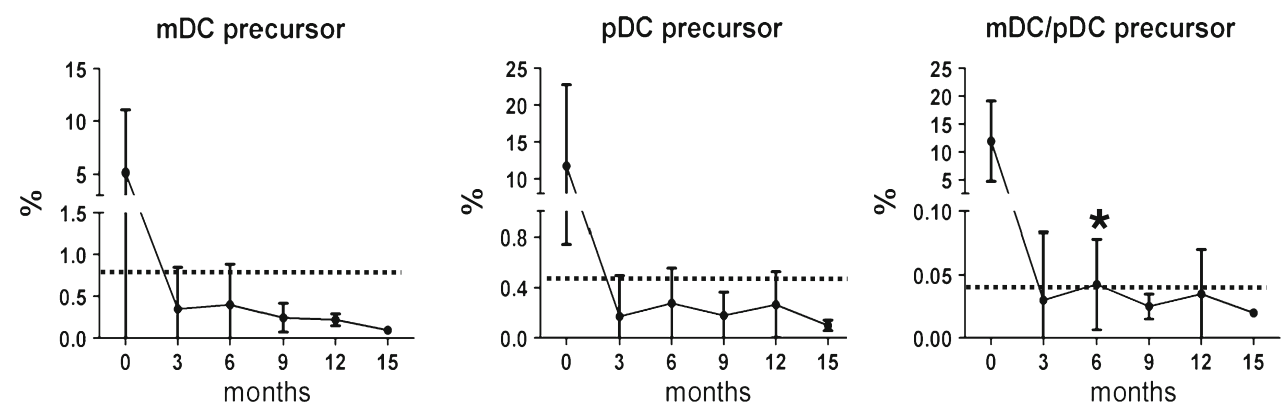

B) Analyses of terminally differentiated DCs prior to relapse
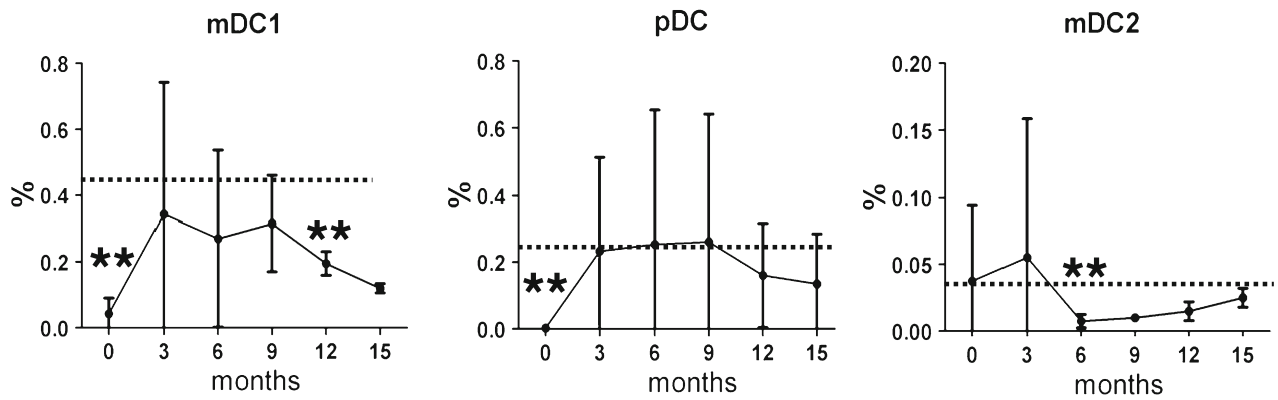
Fig. 5 Secretion of stress cytokines by PBMC samples of CR/REL samples $(n=4)$ was observed as a later event (12 months after FD), yet several months prior to relapse. CR/DFS patients $(n=3)$ were not significantly different to healthy donors (HD, $n=4)$. a Cytokines secreted at 6 months after FD, comparing CR/REL, CR/DFS and HD. b Cytokines secreted at 12 months after FD, comparing CR/REL, CR/DFS, and HD. Single asterisk: significantly lower than CR/DFS $(p<0.05)$. Number sign: trend for lower secretion compared to HD $(p=0.0988)$. [Detection level for cytokines is $3.2 \mathrm{pg} / \mathrm{ml}]$
A) 6 months after FD

IL-10

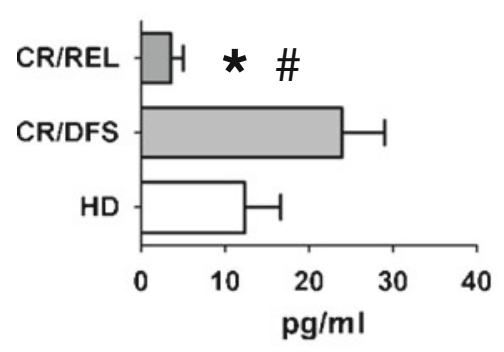

TNF-alpha

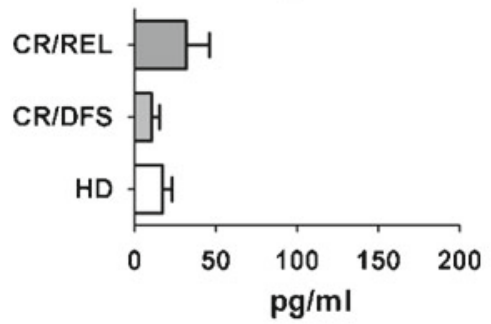

IL-6

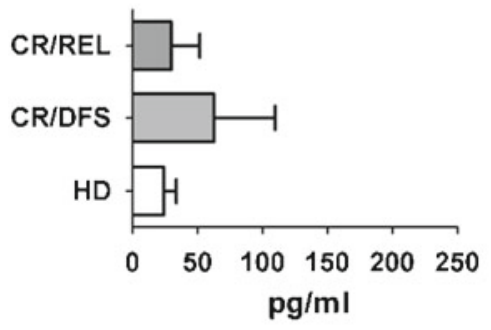

IL-1beta

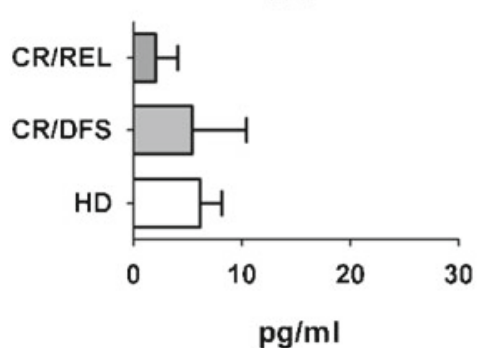

B) 12 months after FD

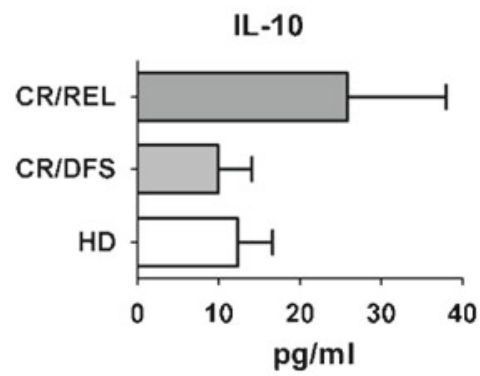

TNF-alpha

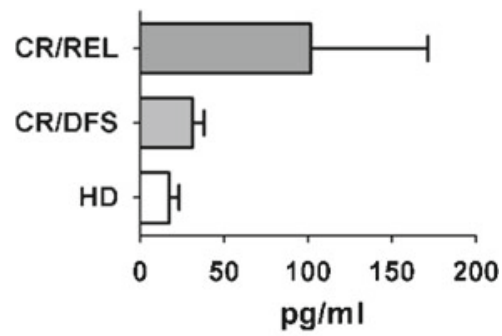

IL-6
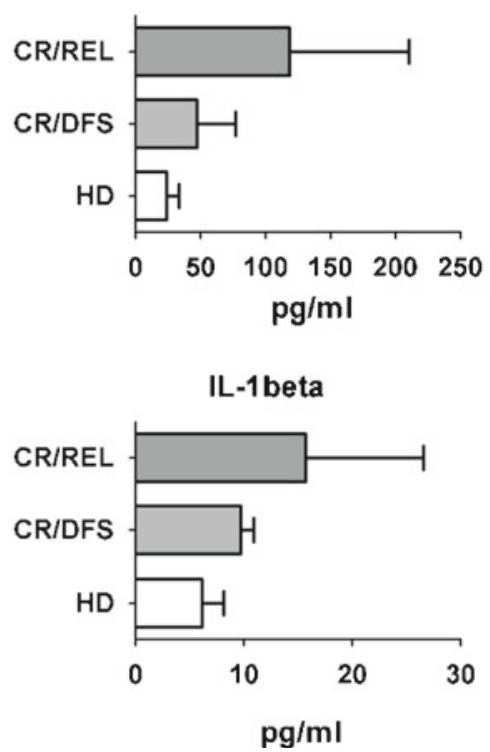

not observe inhibition of DC differentiation (data not shown). We thus evaluated the effects of leukemic cell supernatants on $\mathrm{CD} 14^{+}$monocytes, which are myeloid precursors of mDC. Cell supernatant collected from an ITD $^{+}$ FD sample (obtained from CR/REL patient \#6 (Table 1)) was used as conditioned media for the culture of $\mathrm{CD} 14^{+}$ monocytes obtained from two healthy donors (enriched to 90-95\% purity with immunoconjugated magnetic beads). The experiments were performed using 1:3 and 1:9 leukemic supernatant dilutions in order to evaluate a possible dose-dependent effect. A mock baseline control was run in parallel. The monocytes were collected after 3 days of culture and analyzed for viability and purity, while the supernatants were analyzed for cytokine production. For the two independent experiments, FD leukemia supernatant at 1:3 dilution doubled the viability of monocytes compared to the 1:9 dilution or the mock control (Fig. 6a). Correlating with the increased monocyte viability, the leukemia supernatant (1:3 dilution) induced the secretion of IL-10, TNF- $\alpha$, IL-6, and IL-1 $\beta$ in both experiments (Fig. 6b).

\section{Discussion}

Deregulated dendropoiesis and DC dysfunction have been acknowledged in recent years to trigger immune evasion 
Fig. 6 Soluble factors produced by $\mathrm{ITD}^{+} \mathrm{AML}$ PBMCs (patient \#6) can increase viability and stimulate monocytes to secrete stress cytokines. a ITD ${ }^{+}$AML supernatant increases the viability of monocytes in vitro, as shown on day 3 of culture, and preserves the expression of CD14. b ITD ${ }^{+}$AML supernatant stimulates monocytes to produce stress cytokines after 3 days of co-culture. [Detection level for cytokines is $3.2 \mathrm{pg} / \mathrm{ml}$ ]

\section{A) Effects of ITD+ AML supernatant on monocyte viability and purity}

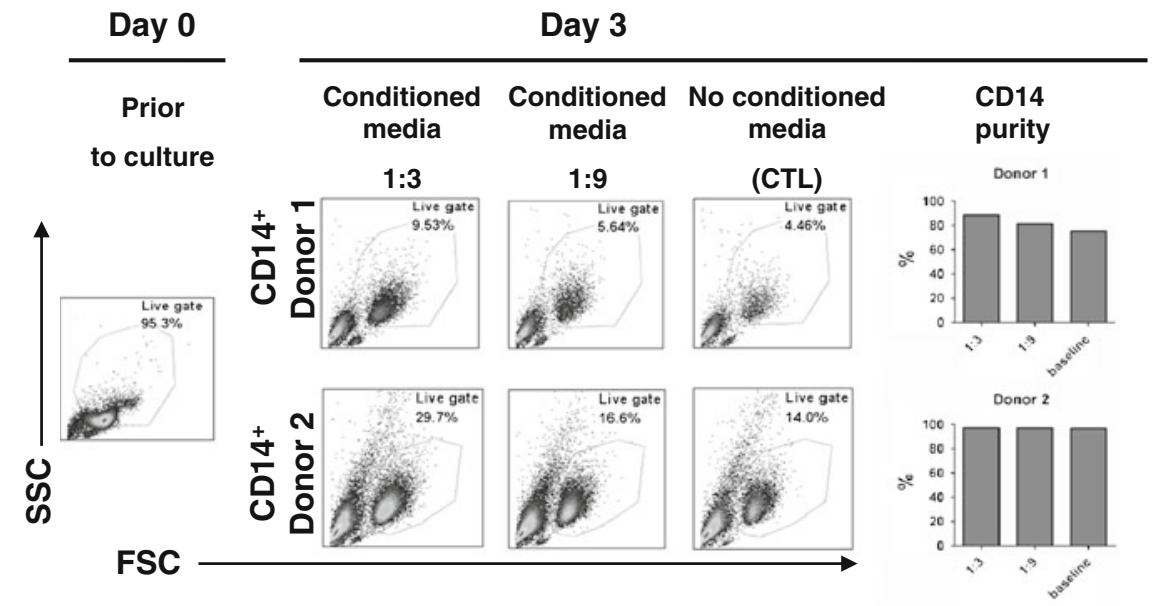

B) Effects of ITD+ AML supernatant on secretion of cytokines by monocytes
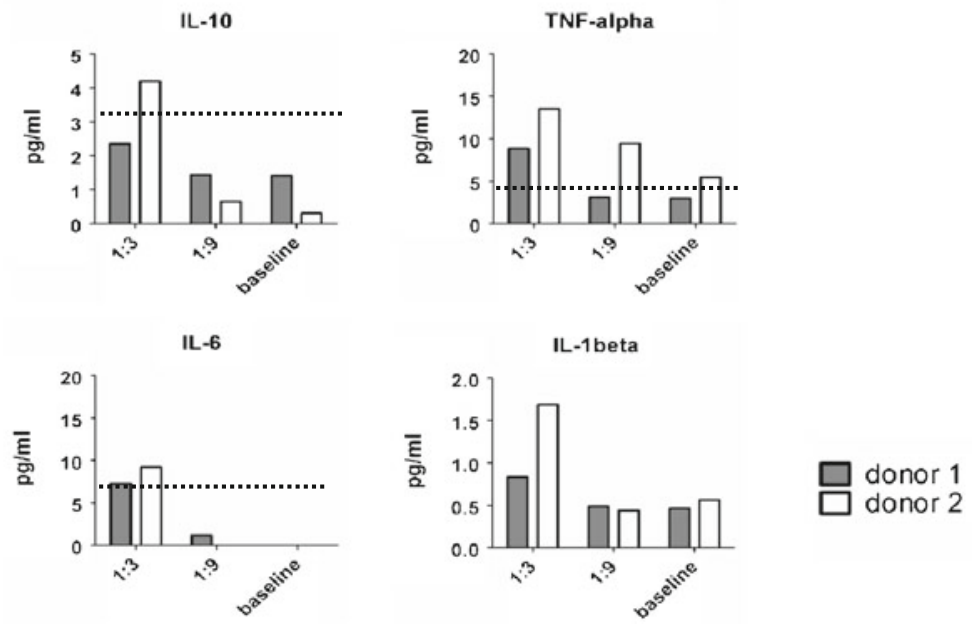

Furthermore, we proposed to evaluate the patterns of dendritic cell lineages and of a subset of immunomodulatory cytokines (IL-10, TNF- $\alpha$, IL-6, and IL-1 $\beta$ ) during the course of ITD $^{+}$leukemia. We obtained samples from patients treated with conventional induction/consolidation chemotherapy, from the first diagnosis to eventual relapse.

Our results indicated an incomplete regeneration of the myeloid dendritic cell compartment in $\mathrm{ITD}^{+}$leukemia patients, which was aggravated prior to relapse (Suppl. Fig. 3). Notably, the decreased frequencies of mDCs in PBMC samples obtained prior to relapse were correlated with increased secretion of IL-10 and stress cytokines (TNF- $\alpha$, IL- 6 , and IL-1 $\beta$ ) in comparison to samples obtained from patients who did not relapse. The fact that leukemic cells could not be detected in these pre-relapse PBMC samples indicated that 


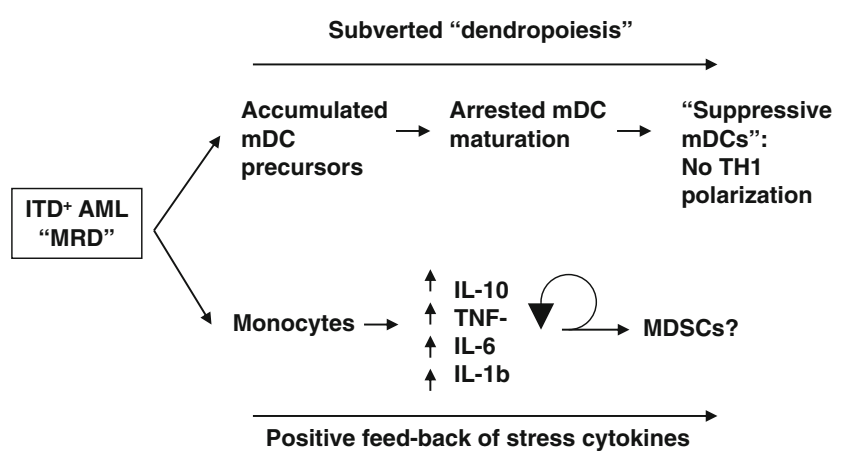

Fig. 7 Proposed model of ITD ${ }^{+}$AML inhibition of DC differentiation and activation of myeloid-derived suppressor cells

non-leukemic cells were involved with secretion of these cytokines. In order to experimentally test this, we demonstrated in a 3-day assay that cell-free supernatants obtained from an ITD $^{+}$diagnostic sample could activate monocytes obtained from healthy donors to secrete IL-10, TNF- $\alpha$, IL-6, and IL$1 \beta$. Based on these observations, we hypothesize that suppression of dendropoiesis during remission of ITD $^{+}$leukemia is mediated by soluble factors (produced by minimal residual leukemia or by the leukemia microenvironment) that activate myeloid cells (such as monocytes) to produce IL-10, IL-6, TNF- $\alpha$, and IL-1 $\beta$. These cytokines in turn could deregulate the differentiation and maturation of myeloid DC (Fig. 7).

IL-6 was defined as a crucial factor for tumor survival and immune evasion, i.e., via induced secretion of IL-10 by regulatory $\mathrm{T}$ cells, which effectively suppresses adaptive immune responses towards tumor antigens [33] and induces proliferation of immature myeloid cells [34]. The combination of IL- 6 and its inducers TNF- $\alpha$ and IL-1 $\beta$ [35] promotes tumor growth and survival in cancer patients through maintenance of chronic inflammation and induction of a tumor-supporting microenvironment $[19,36]$.

Prior to our work, supernatants of FD leukemia samples containing IL-6 and IL-10, TNF- $\alpha$, and IL-1 $\beta$ were shown to trigger a block in DC differentiation in vitro [30]. These results corroborate in part with ours, as we observed high levels of these cytokines only in FD samples of patients who did not enter remission. In our experimental setting, the FD leukemia supernatants did not block ex vivo differentiation of conventional myeloid DC (grown in the presence of GMCSF and IL-4, results not shown). Nevertheless, we were able to show an effect of the FD leukemia supernatants on monocyte viability as a feedback loop for production of these same cytokines. It is important to note that IL- 6 and IL-10, TNF- $\alpha$, and IL-1 $\beta$ have been implied in the generation of the MDSCs, which are commonly found in cancer patients [27-29, 37, 38]. MDSCs are a heterogeneous group of immature myeloid cells arrested in an early differentiation step towards becoming a DC and defined by their immunosuppressive capacities [38-40], which include direct inhibition of tumor-reactive T cells [27, 41]. Interestingly, the ITD mutation itself was shown to be implied in certain regulatory mechanisms, i.e., by induction of increased reactive oxygen species production [42], which is regulating a major mechanism of immune suppression in cancer as induced by MDSCs [37].

Noteworthy, a previous study evaluated serum levels of TNF- $\alpha$, IL-6, and IL-10, in diagnostic samples obtained from patients with AML or high-risk myelodysplastic syndromes. Lower TNF- $\alpha$ levels were found to significantly correlate with better performance status, whereas the other cytokines were not found to be predictive of clinical outcomes [43]. Blockage of TNF- $\alpha$ is already used as standard treatment in various autoimmune diseases [35], whereas clinical trials to evaluate blockage of IL- 6 and IL- $1 \beta$ in inflammatory diseases and cancer are ongoing [35, 44-46]. Thus, evaluation of novel immunomodulatory approaches like these may be also warranted for improving survival of ITD $^{+}$AML patients.

In conclusion, lack of mature $\mathrm{mDCs}$ in $\mathrm{ITD}^{+} \mathrm{FD}$ patient samples in combination with the incomplete regeneration of $\mathrm{mDCs}$ during remission and higher secretion of IL-10 and stress cytokines (TNF- $\alpha$, IL-6, and IL$1 \beta)$ in patients who relapsed strengthen the hypothesis of immunomodulatory changes during the course of $\mathrm{ITD}^{+}$ AML relapse. These events are likely to be linked with a "subverted dendropoiesis" and induction of MDSC in these leukemia patients (Fig. 7). Thus, clinical monitoring of PBMC samples of $\mathrm{ITD}^{+}$patients during remission in order to enumerate the DC profile and to detect the secretion of IL-10 and stress cytokine levels as well as the occurrence of MDSCs might serve as immunologic biomarkers to provide additional approaches for disease staging.

Acknowledgments We thank the members of the Stripecke laboratory for their contributions to the completion of this work. We thank the clinicians, nurses, and technicians in the Department of Hematology, Hemostasis, Oncology and Stem Cell Transplantation for assistance with the sample collections and molecular analyses for establishing a cell bank for these studies (diagnosis, remission, and relapse samples). We thank the AML Study Group for access to additional first diagnosis samples. Use of the LSR II flow cytometer was made possible through SFB-738. This work was supported by a collaborative research grant from the Jose Carreras Foundation (to R.S.), a grant of the German Research Council (DFG/SFB738 to R.S.), by the DFG Excellence Cluster Rebirth in Regenerative Medicine (to R.S.) and by the Deutsche Krebshilfe (to R.S.). M.R. was supported by a MHHHBRS/Strucmed fellowship.

Conflict of interest The authors declare that they have no conflict of interest.

Open Access This article is distributed under the terms of the Creative Commons Attribution License which permits any use, distribution, and reproduction in any medium, provided the original author(s) and the source are credited. 


\section{References}

1. Estey EH (2012) Acute myeloid leukemia: 2012 update on diagnosis, risk stratification, and management. Am J Hematol 87(1):89-99. doi:10.1002/ajh.22246

2. Maraskovsky E, Daro E, Roux E, Teepe M, Maliszewski CR, Hoek J, Caron D, Lebsack ME, McKenna HJ (2000) In vivo generation of human dendritic cell subsets by Flt3 ligand. Blood 96(3):878-884

3. Li L, Piloto O, Kim KT, Ye Z, Nguyen HB, Yu X, Levis M, Cheng L, Small D (2007) FLT3/ITD expression increases expansion, survival and entry into cell cycle of human haematopoietic stem/ progenitor cells. Br J Haematol 137(1):64-75. doi:10.1111/j.13652141.2007.06525.x

4. Gale RE, Green C, Allen C, Mead AJ, Burnett AK, Hills RK, Linch DC (2008) The impact of FLT3 internal tandem duplication mutant level, number, size, and interaction with NPM1 mutations in a large cohort of young adult patients with acute myeloid leukemia. Blood 111(5):2776-2784. doi:10.1182/blood-2007-08109090

5. Schlenk RF, Dohner K, Krauter J, Frohling S, Corbacioglu A, Bullinger L, Habdank M, Spath D, Morgan M, Benner A, Schlegelberger B, Heil G, Ganser A, Dohner H (2008) Mutations and treatment outcome in cytogenetically normal acute myeloid leukemia. N Engl J Med 358(18):1909-1918. doi:10.1056/ NEJMoa074306

6. Heuser M, Beutel G, Krauter J, Dohner K, von Neuhoff N, Schlegelberger B, Ganser A (2006) High meningioma 1 (MN1) expression as a predictor for poor outcome in acute myeloid leukemia with normal cytogenetics. Blood 108(12):3898-3905. doi:10.1182/blood-2006-04-014845

7. Marcucci G, Haferlach T, Dohner H (2011) Molecular genetics of adult acute myeloid leukemia: prognostic and therapeutic implications. J Clin Oncol 29(5):475-486. doi:10.1200/JCO.2010.30.2554

8. Thol F, Kolking B, Damm F, Reinhardt K, Klusmann JH, Reinhardt D, von Neuhoff N, Brugman MH, Schlegelberger B, Suerbaum S, Krauter J, Ganser A, Heuser M (2012) Next-generation sequencing for minimal residual disease monitoring in acute myeloid leukemia patients with FLT3-ITD or NPM1 mutations. Gene Chromosome Cancer. doi:10.1002/gcc.21955

9. Brunet S, Labopin M, Esteve J, Cornelissen J, Socie G, Iori AP, Verdonck LF, Volin L, Gratwohl A, Sierra J, Mohty M, Rocha V (2012) Impact of FLT3 internal tandem duplication on the outcome of related and unrelated hematopoietic transplantation for adult acute myeloid leukemia in first remission: a retrospective analysis. J Clin Oncol 30(7):735-741. doi:10.1200/JCO.2011.36.9868

10. Yan CH, Liu DH, Liu KY, Xu LP, Liu YR, Chen H, Han W, Wang Y, Qin YZ, Huang XJ (2012) Risk stratification-directed donor lymphocyte infusion could reduce relapse of standard-risk acute leukemia patients after allogeneic hematopoietic stem cell transplantation. Blood 119(14):3256-3262. doi:10.1182/blood-201109-380386

11. Banchereau J, Steinman RM (1998) Dendritic cells and the control of immunity. Nature 392(6673):245-252. doi:10.1038/32588

12. Palucka K, Banchereau J (2012) Cancer immunotherapy via dendritic cells. Nat Rev Cancer 12(4):265-277. doi:10.1038/nrc3258

13. Almand B, Resser JR, Lindman B, Nadaf S, Clark JI, Kwon ED, Carbone DP, Gabrilovich DI (2000) Clinical significance of defective dendritic cell differentiation in cancer. Clin Cancer Res 6(5):1755-1766

14. Rickmann M, Krauter J, Stamer K, Heuser M, Salguero G, Mischak-Weissinger E, Ganser A, Stripecke R (2011) Elevated frequencies of leukemic myeloid and plasmacytoid dendritic cells in acute myeloid leukemia with the FLT3 internal tandem duplication. Ann Hematol. doi:10.1007/s00277-011-1231-2
15. Elenkov IJ, Chrousos GP (1999) Stress hormones, Th1/Th2 patterns, pro/anti-inflammatory cytokines and susceptibility to disease. Trends Endocrinol Metab TEM 10(9):359-368

16. Elenkov IJ, Chrousos GP (2002) Stress hormones, proinflammatory and antiinflammatory cytokines, and autoimmunity. Ann N Y Acad Sci 966:290-303

17. Sato T, Terai M, Tamura Y, Alexeev V, Mastrangelo MJ, Selvan SR (2011) Interleukin 10 in the tumor microenvironment: a target for anticancer immunotherapy. Immunol Res 51(2-3):170-182. doi:10.1007/s12026-011-8262-6

18. Mossner R, Mikova O, Koutsilieri E, Saoud M, Ehlis AC, Muller N, Fallgatter AJ, Riederer P (2007) Consensus paper of the WFSBP Task Force on Biological Markers: biological markers in depression. World J Biol Psychiatry 8(3):141-174. doi:10.1080/ 15622970701263303

19. Multhoff G, Molls M, Radons J (2011) Chronic inflammation in cancer development. Front Immunol 2:98. doi:10.3389/ fimmu.2011.00098

20. Wu H, Li P, Shao N, Ma J, Ji M, Sun X, Ma D, Ji C (2012) Aberrant expression of Treg-associated cytokine IL-35 along with IL-10 and TGF-beta in acute myeloid leukemia. Oncol Lett 3(5):1119-1123. doi:10.3892/ol.2012.614

21. Frohling S, Schlenk RF, Breitruck J, Benner A, Kreitmeier S, Tobis K, Dohner H, Dohner K (2002) Prognostic significance of activating FLT3 mutations in younger adults (16 to 60 years) with acute myeloid leukemia and normal cytogenetics: a study of the AML Study Group Ulm. Blood 100(13):4372-4380. doi:10.1182/ blood-2002-05-1440

22. Weisser M, Kern W, Rauhut S, Schoch C, Hiddemann W, Haferlach T, Schnittger S (2005) Prognostic impact of RT-PCRbased quantification of WT1 gene expression during MRD monitoring of acute myeloid leukemia. Leukemia 19(8):1416-1423. doi:10.1038/sj.leu.2403809

23. Mosser DM, Zhang X (2008) Interleukin-10: new perspectives on an old cytokine. Immunol Rev 226:205-218. doi:10.1111/j.1600065X.2008.00706.X

24. Burger D, Dayer JM (2002) Cytokines, acute-phase proteins, and hormones: IL-1 and TNF-alpha production in contact-mediated activation of monocytes by $\mathrm{T}$ lymphocytes. Ann N Y Acad Sci 966:464-473

25. Talarn C, Urbano-Ispizua A, Martino R, Perez-Simon JA, Batlle M, Herrera C, Granell M, Gaya A, Torrebadell M, Fernandez-Aviles F, Aymerich M, Marin P, Sierra J, Montserrat E (2007) Kinetics of recovery of dendritic cell subsets after reduced-intensity conditioning allogeneic stem cell transplantation and clinical outcome. Haematologica 92(12):1655-1663. doi:10.3324/haematol.11076

26. Porta MD, Rigolin GM, Alessandrino EP, Maiocchi M, Malcovati L, Vanelli L, Barate C, Rumi E, Ciccone M, Cuneo A, Lazzarino M, Castoldi G (2004) Dendritic cell recovery after allogeneic stemcell transplantation in acute leukemia: correlations with clinical and transplant characteristics. Eur J Haematol 72(1):18-25. doi:10.1046/j.0902-4441.2004.00172.x

27. Condamine T, Gabrilovich DI (2011) Molecular mechanisms regulating myeloid-derived suppressor cell differentiation and function. Trends Immunol 32(1):19-25. doi:10.1016/j.it.2010.10.002

28. Nagaraj S, Schrum AG, Cho HI, Celis E, Gabrilovich DI (2010) Mechanism of $\mathrm{T}$ cell tolerance induced by myeloid-derived suppressor cells. J Immunol 184(6):3106-3116. doi:10.4049/ jimmunol.0902661

29. Cheng P, Corzo CA, Luetteke N, Yu B, Nagaraj S, Bui MM, Ortiz M, Nacken W, Sorg C, Vogl T, Roth J, Gabrilovich DI (2008) Inhibition of dendritic cell differentiation and accumulation of myeloid-derived suppressor cells in cancer is regulated by S100A9 protein. J Exp Med 205(10):2235-2249. doi:10.1084/jem.20080132

30. Motta JM, Nascimento CR, Rumjanek VM (2010) Leukemic cell products down-regulate human dendritic cell differentiation. 
Cancer Immunol Immunother 59(11):1645-1653. doi:10.1007/ s00262-010-0890-5

31. Almand B, Clark JI, Nikitina E, van Beynen J, English NR, Knight SC, Carbone DP, Gabrilovich DI (2001) Increased production of immature myeloid cells in cancer patients: a mechanism of immunosuppression in cancer. J Immunol 166(1):678-689

32. Pinzon-Charry A, Maxwell T, Lopez JA (2005) Dendritic cell dysfunction in cancer: a mechanism for immunosuppression. Immunol Cell Biol 83(5):451-461. doi:10.1111/j.1440-1711.2005.01371.x

33. Ara T, Declerck YA (2010) Interleukin-6 in bone metastasis and cancer progression. Eur J Cancer 46(7):1223-1231. doi:10.1016/ j.ejca.2010.02.026

34. Leung KN, Mak NK, Fung MC (2005) Cytokines in the differentiation therapy of leukemia: from laboratory investigations to clinical applications. Crit Rev Clin Lab Sci 42(5-6):473-514. doi:10.1080/ 10408360500295154

35. Moller B, Villiger PM (2006) Inhibition of IL-1, IL-6, and TNFalpha in immune-mediated inflammatory diseases. Springer Semin Immunopathol 27(4):391-408. doi:10.1007/s00281-006-0012-9

36. Multhoff G, Radons J (2012) Radiation, inflammation, and immune responses in cancer. Front Oncol 2:58. doi:10.3389/ fonc. 2012.00058

37. Corzo CA, Cotter MJ, Cheng P, Cheng F, Kusmartsev S, Sotomayor E, Padhya T, McCaffrey TV, McCaffrey JC, Gabrilovich DI (2009) Mechanism regulating reactive oxygen species in tumor-induced myeloid-derived suppressor cells. J Immunol 182(9):5693-5701. doi:10.4049/jimmunol.0900092

38. Gabrilovich DI, Nagaraj S (2009) Myeloid-derived suppressor cells as regulators of the immune system. Nat Rev Immunol 9(3):162-174. doi:10.1038/nri2506

39. Filipazzi P, Huber V, Rivoltini L (2012) Phenotype, function and clinical implications of myeloid-derived suppressor cells in cancer patients. Cancer Immunol Immunother 61(2):255-263. doi:10.1007/s00262-011-1161-9

40. Bunt SK, Yang L, Sinha P, Clements VK, Leips J, Ostrand-Rosenberg $S$ (2007) Reduced inflammation in the tumor microenvironment delays the accumulation of myeloid-derived suppressor cells and limits tumor progression. Cancer Res 67(20):10019-10026. doi:10.1158/00085472.CAN-07-2354

41. Mougiakakos D, Jitschin R, von Bahr L, Poschke I, Gary R, Sundberg B, Gerbitz A, Ljungman P, Le Blanc K (2012) Immunosuppressive CD14(+)HLA-DR(low/neg) IDO(+) myeloid cells in patients following allogeneic hematopoietic stem cell transplantation. Leukemia. doi:10.1038/leu.2012.215

42. Sallmyr A, Fan J, Datta K, Kim KT, Grosu D, Shapiro P, Small D, Rassool F (2008) Internal tandem duplication of FLT3 (FLT3/ITD) induces increased ROS production, DNA damage, and misrepair: implications for poor prognosis in AML. Blood 111(6):31733182. doi:10.1182/blood-2007-05-092510

43. Tsimberidou AM, Estey E, Wen S, Pierce S, Kantarjian H, Albitar M, Kurzrock R (2008) The prognostic significance of cytokine levels in newly diagnosed acute myeloid leukemia and high-risk myelodysplastic syndromes. Cancer 113(7):1605-1613. doi:10.1002/cncr.23785

44. Liu Y, Li PK, Li C, Lin J (2010) Inhibition of STAT3 signaling blocks the anti-apoptotic activity of IL-6 in human liver cancer cells. J Biol Chem 285(35):27429-27439. doi:10.1074/jbc.M110.142752

45. Ferretti E, Di Carlo E, Cocco C, Ribatti D, Sorrentino C, Ognio E, Montagna D, Pistoia V, Airoldi I (2010) Direct inhibition of human acute myeloid leukemia cell growth by IL-12. Immunol Lett 133(2):99-105. doi:10.1016/j.imlet.2010.08.002

46. de Mejia EG, Wang W, Dia VP (2010) Lunasin, with an arginineglycine-aspartic acid motif, causes apoptosis to L1210 leukemia cells by activation of caspase-3. Mol Nutr Food Res 54(3):406414. doi:10.1002/mnfr.200900073 